\title{
Antibacterial and Cytotoxicity Analyses on Aloe Vera Body Soap
}

\author{
Alex Zhen Kai Loํㅜ, Nurizzati Mohd Daud ${ }^{1}$, Norhidayu Muhamad Zain²* \\ ${ }^{1}$ School of Biomedical Engineering and Health Sciences, Universiti Teknologi Malaysia, 81310 UTM Johor Bahru, \\ Johor, Malaysia. \\ ${ }^{2}$ Academy of Islamic Civilization, Faculty of Social Sciences and Humanities, Universiti Teknologi Malaysia, \\ 81310 UTM Johor Bahru, Johor, Malaysia \\ *Corresponding Author norhidayu_mz@utm.my
}

Received 2 December 2021; Accepted 17 January 2022; Available 31 Jauary 2022

https://doi.org/10.11113/humentech/v1n1.13

\begin{abstract}
:
Aloe vera is a well-known succulent plant and it is often used to moisturise and sooth the skin. In this study, Aloe vera is incorporated into natural soap to investigate the antibacterial properties and cytotoxicity of the samples. The antibacterial properties of the Aloe vera sample was determined by performing zone inhibition and bacterial colony test on Escherichia coli (E. coli). The cell viability test was conducted with human skin fibroblast cells through MTT assay. The Aloe vera samples showed antibacterial properties through the presence of inhibition zones and in the bacterial colony analysis, the Aloe vera samples have inhibitory effect against $E$. coli with a bacterial retardation percentage of $31.97 \pm 0.90 \%$. The cell viability percentage of $95.30 \pm 11.16 \%$ was found on the Aloe vera sample with normal cell morphology. These results testified that the Aloe vera sample was biocompatible and possessed antibacterial properties which is suitable to be incorporated in skin products.
\end{abstract}

Keywords: Aloe vera; Soap; Antibacterial; Biocompatible

\section{Introduction}

Natural medicinal resources are commonly been emphasised in the application of skin care and skin treatment. Previous studies have scientifically showed medicinal plants such as Aloe vera [1], Achyranthes aspera [2], Centella asiatica (centella) [3] and Actinidia deliciosa (kiwifruit) [4] have an ability to enhance and accelerate the healing process of burn wound. Aloe vera is a type of succulent plant that grows naturally in tropical climate around the world. Aloe vera gel is commonly used in treating burn wound, tissue sore, tissue scar and inflammation [1]. It is also being embedded inside cosmetic products for its moisturising and soothing effects [5]. Besides, Aloe vera is effective in managing pigmentation, stretch marks and wrinkles [6]. Its mechanisms of action are mostly based on the stimulation of collagen, fibroblast and macrophages for the proliferation of connective tissues and phagocytosis activation [5]. Furthermore, Aloe vera is pre-scientifically proved to have an antibacterial property by retarding the growth of several skin pathogens such as Staphylococcus aureus, Staphylococcus epidermidis, Escherichia coli, Klebsiella pneumoniae, Pseudomonas aeruginosa, Proteus vulgaris and Proteus mirabilis [7].

In this study, Aloe vera was incorporated into soap through a saponification process. Saponification is a costeffective process which does not requires high-energy consumption and dangerous chemical reagents. It employed the counter current mixing of fatty acids and triglyceride in producing bar soap for washing and cleaning purposes [8]. The incorporation of Aloe vera is intended to equip a soap with biocompatible and antibacterial properties to be used on the skin. Therefore, this study aimed to investigate the antibacterial and biocompatibility properties of naturally derived soap 
consisted of Aloe vera. The samples were prepared using a natural cold process. The antibacterial property was evaluated against Escherichia coli (E. coli) through zone inhibition test and bacterial colony test. While, the cytocompatibility property was determined with human skin fibroblast cells through MTT assay and morphology observation under an inverted microscope.

\section{Materials and Methods}

\subsection{Sample preparation}

Aloe vera soap was obtained from Pure Natural Resources Sdn. Bhd., Malaysia. A cold process method was used to prepare the samples by mixing $75 \% \mathrm{v} / \mathrm{v}$ fixed oils, $5 \% \mathrm{v} / \mathrm{v}$ sodium hydroxide $(\mathrm{NaOH})$ and $20 \%$ v/v Aloe vera extracts. The fixed oils were composed of 50\% extra virgin olive oil, $15 \%$ Aloe vera oil, $15 \%$ shea butter, $10 \%$ unrefined avocado oil, $7 \%$ cold press extra virgin coconut oil and $3 \%$ neem oil.

\subsection{Antibacterial test}

Gram-negative bacteria, Escherichia coli (ATCC 25922) was used to investigate the antibacterial property of the samples. The Aloe vera soap were pre-evaluated through a zone inhibition test or also known as a disc diffusion technique. The samples were punched into a disc shape with a diameter of $10 \mathrm{~mm}$ and $5 \mathrm{~mm}$ and sterilised using $70 \%$ ethanol solution. The sterilised discs were then placed on Luria-Bertani (LB) agar that has been smeared with bacteria. The discs were given soft pressure to fix its position and incubated in an incubator at $37^{\circ} \mathrm{C}$ for 24 hours. The inhibition zones of the discs towards $E$. coli were observed. The analysis was carried out in triplicate and the average data were calculated.

The antibacterial property of the samples was further evaluated through a bacterial colony test by calculating the percentage of antibacterial efficacy. The Aloe vera samples were cut into small cubes with a weight of $0.01 \mathrm{~g}$. The cubes were washed with $70 \%$ ethanol for sterilisation purpose. Each cube was then dissolved in $5 \mathrm{~mL}$ distilled water and vortexed for 30 seconds to acquire the extraction suspension. The bacteria with a concentration of $1 \times 10^{6}$ cells $/ \mathrm{mL}$ were pipetted into the extraction suspension and incubated in a shaking incubator for 3 hours at $200 \mathrm{rpm}$ with a temperature of $37^{\circ} \mathrm{C}$. Two-fold dilution series of bacterial suspension were performed in deionised water at a ratio of 1:9. Later, 10 $\mu \mathrm{L}$ of each dilution series was drop on LB agar and incubated at $37^{\circ} \mathrm{C}$ for 24 hours. The bacterial colonies formed on the agar were counted to obtain the percentage of antibacterial efficacy [9]. The control for the bacterial colony test was set to the addition of $1 \mathrm{~mL}$ of $70 \%$ ethanol in the extraction suspension without the introduction of sample.

\subsection{Cytotoxicity test}

The biocompatibility of the samples was investigated through a cytotoxicity test with human skin fibroblast cells (HSF 1184). The viability of cell was assessed by MTT assay. The MTT assay was based on the ability of live cells to reduce a tetrazolium-based compound (MTT) into a purplish formazan product. The extraction suspension of the samples were prepared following American Society for Testing and Materials F813-07 (ASTM F813-07) by immersing 0.01 g cube soap in $1 \mathrm{~mL}$ Dulbecco's Modified Eagle Medium (DMEM). Initially, the HSF cells were sub-cultured in DMEM supplemented with $10 \%$ (v/v) foetal bovine serum (FBS) (Gibco, USA) and 1\% penicillin/streptomycin. At passage four to five, $1 \times 10^{5} \mathrm{HSF}$ cells were allowed to attach on a 96 well-plate for 24 hours. The extraction suspension were then pipetted in each well and further incubated for 24 hours at $37^{\circ} \mathrm{C}$ with the presence of $5 \%$ carbon dioxide $\left(\mathrm{CO}_{2}\right)$. The cell morphology was then observed under an inverted microscope (AX10, Zeiss, Germany) at a magnification of 20×.

The cells were then washed with phosphate buffered saline (PBS) to remove non-attached cells. A total of $200 \mu \mathrm{L}$ MTT $(5 \mathrm{mg} / \mathrm{mL})$ solution was added to each well and incubated for 4 hours to allow a complete reaction. After 4 hours, the solution were discarded and $150 \mu \mathrm{L}$ dimethyl sulfoxide (DMSO) was pipetted into each well to dissolve the formazan crystal. The formazan products were measured at $540 \mathrm{~nm}$ wavelength using a microplate reader (Thermo Scientific, USA). Finally, the procedure of cytotoxicity test was repeated without immersion of the sample in the extraction suspension as the control sample. All analyses were carried out in triplicate and the data were presented as mean \pm standard deviation.

\section{Results \& Discussion}

\subsection{Antibacterial analysis}

The Aloe vera samples show inhibition zones on E. coli as shown in Figure. 1(A) indicating its antibacterial properties. Wide inhibition zones produced by the Aloe vera samples clearly testified its antibacterial effectiveness. Although the antibacterial activity of Aloe vera was low, it was suggested that the addition of the compound as an ingredient in any antibacterial product may favour direct antibacterial effect on accessible areas of the body [6,7]. The 
distances of the inhibition zones were not recorded due to indistinct inhibition zones as the samples were slightly diluted within the agar.

The results of inhibition zones were further verified with the bacterial colony analysis. Figure. 1(B) shows percentage of antibacterial efficacy of natural Aloe vera soap. The Aloe vera samples have inhibitory effect against $E$. coli with an antibacterial percentage of $31.97 \pm 0.90 \%$. In comparison, the control sample that consisted of $70 \%$ ethanol possessed $100 \%$ antibacterial percentage. These results are in accordance to the results of the inhibition zones, clarifying the effectiveness of the Aloe vera samples in retarding the growth of E. coli.

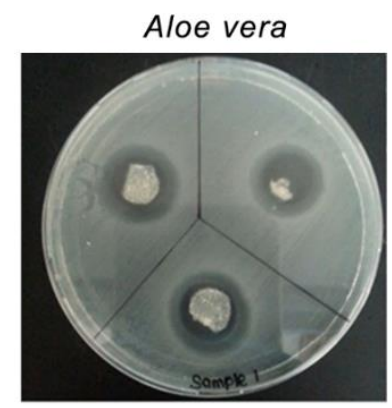

(A)

(B)

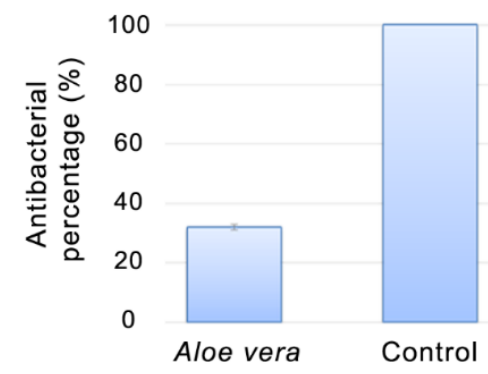

Figure 1. Inhibition zones (A) and antibacterial percentage (B) on Aloe vera soap

The antibacterial capability of Aloe vera soap is mostly contributed by the phytochemical components that existed in the Aloe vera which permit the effect to kill E. coli. Specific Aloe vera compounds that involves in the antibacterial activity of Aloe vera are anthraquinones and dihydroxyanthraquinones as well as saponins. Bradford and Awad [10] have proposed Aloe vera to have direct antimicrobial activity. Other than that, phytosterols have also been shown to directly inhibit tumour growth by slowing cell cycle progression through induction of apoptosis and inhibition of tumour metastasis [10]. Additionally, phytosterols also can reduce biomarkers for oxidative stress and inflammation [11] and reduce cancer development by enabling antitumor responses, increasing immune recognition of cancer, influencing hormonal-dependent growth of endocrine tumours and altering sterol biosynthesis [11].

\subsection{Cytotoxicity analysis}

The Aloe vera samples did not cause cell retardation when tested on human skin fibroblast cells. The cell viability showed $95.30 \pm 11.16 \%$ on the Aloe vera soap (Figure 2) suggesting its compatibility to be used on the skin. Boudreau and Beland [12] mentioned that Aloe vera gel has displayed non-toxic and may stimulate cellular proliferation even at $100 \%$ concentration.

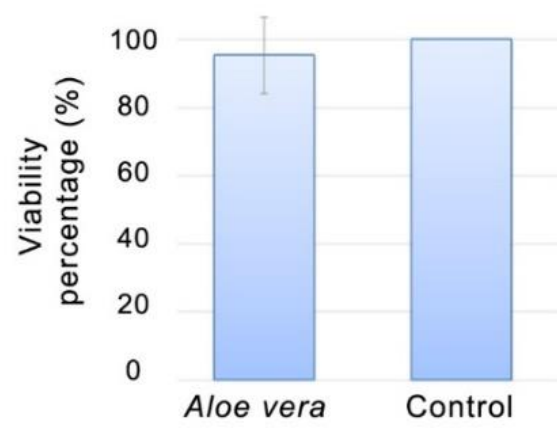

Figure 2. Viability percentage

Besides, in this study, the HSF cells exhibited normal morphologies in accordance to the control when the cells were in contact with the Aloe vera (Figure 3). There was no sign of ruptured morphology even there was some uptake of Aloe vera particles by the cells. However, it did not affect the cell viability as the Aloe vera could improve wound healing and inhibit inflammation [13]. 


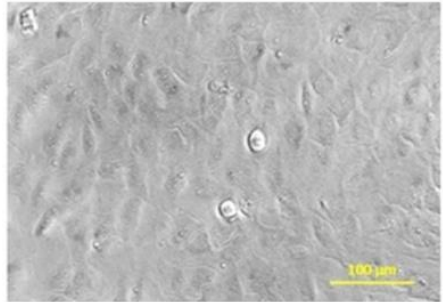

Control

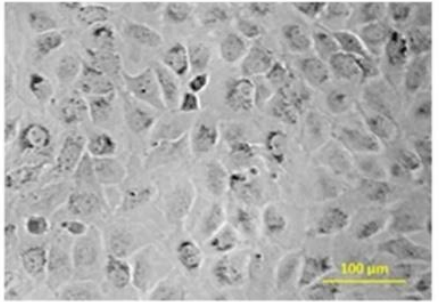

Aloe vera

Figure 3. Morphologies of control and Aloe vera soap

\section{Conclusion}

The antibacterial and cell viability analyses showed that the Aloe vera soap has antibacterial properties and biocompatible with human skin cells. The soap is suitable to be used on human skin which might be able to control inflammation and infection while soothing the skin.

\section{Acknowledgment}

This study was supported by Malaysian Ministry of Higher Education through Research University Grant (RUG) Tier 2 [Q.J130000.2653.16J05].

\section{References}

[1] M. N. Shahzad and N. Ahmed, Effectiveness of Aloe vera gel compared with 1\% silver sulphadiazine cream as burn wound dressing in second degree burns, Journal of the Pakistan Medical Association, 2013, 63(2):225-230.

[2] C. C. Barua, A. Talukdar, A., S. A. Begum, D. C. Pathak, D. K. Sarma, R. S. Borah and A. Gupta, In vivo wound healing efficacy and antioxidant activity of Achyranthes aspera in experimental burns, Pharmaceutical Biology, 2012, 50(7):892-899. https://doi.org/10.3109/13880209.2011.642885

[3] J. Somboonwong, M. Kankaisre, B. Tantisira and M. H. Tantisira, Wound healing activities of different extracts of Centella asiatica in incision and burn wound models: An experimental animal study, BMC Complementary and Alternative Medicine, 2012, 12:103. https://dx.doi.org/10.1186\%2F1472-6882-12-103

[4] F. Hafezi, F., H. E. Rad, B. Naghibzadeh, A. Nouhi and G Naghibzadeh, Actinidia deliciosa (kiwifruit), a new drug for enzymatic debridement of acute burn wounds, Burns: Journal of the International Society for Burn Injuries, 2010, 36(3):352-355. https://doi.org/10.1016/j.burns.2009.04.021

[5] O. Grundmann, Aloe vera gel research review. An overview of its clinical uses and proposed mechanisms of action, Natural Medicine Journal, 2012, 4(9).

[6] N. Tabassum and M. Hamdani, Plants used to treat skin diseases, Pharmacognosy Reviews, 2014, 8(15):52-60. https://doi.org/10.4103/0973-7847.125531.

[7] M. Udgire and G. Pathade, Antibacerial activity of Aloe vera against skin pathogens, American Journal of Ethnomedicine, 2014, 1(3):147-151.

[8] N. P. Vidal, O. A. Adigun, T. H. Pham, A. Mumtaz, C. Manful, G. Callahan, P. Stewart, D. Keough and R. H. Thomas, The effects of cold saponification on the unsaponified fatty acid composition and sensory perception of commercial natural herbal soaps, Molecules, 2008, 23(9):2356.

https://dx.doi.org/10.3390\%2Fmolecules23092356

[9] N. M. Daud, I. F. S. Bahri, N. A. N. N. Malek, H. Hermawan and S. Saidin, Immobilization of antibacterial chlorhexidine on stainless steel using crosslinking polydopamine film: Towards infection resistant medical devices, Colloids and Surfaces. B, Biointerfaces, 2016, 145:130-139. https://doi.org/10.1016/j.colsurfb.2016.04.046

[10] P. G. Bradford and A. B. Awad, Phytosterols as anticancer compounds, Molecular Nutrition and Food Research, 2007, 51(2):161-170. https://doi.org/10.1002/mnfr.200600164

[11] S. Devaraj and I. Jialal, The role of dietary supplementation with plant sterols and stanols in the prevention of cardiovascular disease, Nutrition Reviews, 2006, 64(7):348-354. https://doi.org/10.1111/j.17534887.2006.tb00219.x

[12] M. D. Boudreau and F.A. Beland, An evaluation of the biological and toxicological properties of Aloe barbadensis (miller), Aloe vera, Journal of Environmental Science and Health Part C, 2006, 24(1):103-154. https://doi.org/10.1080/10590500600614303 
[13] R. H. Davis, R.H., J. Donato, G. M. Hartman and R. C. Haas, Anti-inflammatory and wound healing activity of a growth substance in Aloe vera, Journal of the American Podiatric Medical Association, 1994, 84(2):77-81. https://doi.org/10.7547/87507315-84-2-77 